\title{
Pure stress urinary incontinence: analysis of prevalence, estimation of costs, and financial impact
}

Emanuele Rubilotta ${ }^{*}$, Matteo Balzarro ${ }^{1}$, Antonio D'Amico ${ }^{1}$, Maria Angela Cerruto ${ }^{1}$, Silvia Bassi ${ }^{1}$, Chiara Bovo ${ }^{2}$, Valerio lacovelli ${ }^{3}$, Daniele Bianchi ${ }^{3}$, Walter Artibani ${ }^{1}$ and Enrico Finazzi Agrò ${ }^{3}$

\begin{abstract}
Background: The prevalence of pure stress urinary incontinence (P-SUI) and the role of urodynamic investigation (UDI) prior to surgery for stress urinary incontinence (SUI) is debated. Since the exact prevalence of P-SUI is not clear, its clinical and economic impact is not well defined. The aims of this study were to evaluate the prevalence of P-SUI in a population of women who underwent UDI for urinary incontinence (UI), also assessing: 1) the correspondence between clinical diagnosis of P-SUI and urodynamic findings; 2) the analysis of costs in terms of UDI and eventually post-UDI avoided surgical procedures.
\end{abstract}

Methods: A single cohort of women who underwent UDI for UI between January 2012 and July 2016 was prospectively collected and retrospectively analyzed. Clinical P-SUI was defined by the strict criteria of the International Continence Society. For each patient, history, physical examination and UDI were collected. The correspondence between clinical and urodynamic findings of P-SUI was analyzed. The rate of clinical P-SUI changed after performing UDI and the number of unnecessary intervention after UDI were reported. A wide cost analysis of UDIs, and the amount of surgical procedures that were believed unnecessary after UDI was reported.

Results: Stress urinary incontinence was present in 323/544 (59.4\%) patients. The prevalence of clinical P-SUI was 20.7\% (67/323), while the prevalence of complicated SUI (C-SUI) was 79.3\% (256/323). After UDI, diagnosis of P-SUI decreased to $18.3 \%$ (59/232). In 10.2\% of cases (6/59) the scheduled middle urethral sling (MUS) was suppressed after the UDI results because 3/6 cases had detrusor overactivity and urge incontinence, in 2/6 cases SUI was treated with a conservative management, in 1/6 case an important voiding dysfunction was detected. Considering the national reimbursement in our country, the cost of each UDI was 296.5 euros and the total amount was 17,493.5 euros. So far the surgery-related savings covered $61.7-105.0 \%$ of the costs of total number of UDls performed in the uncomplicated patients.

Conclusions: The prevalence of clinical P-SUI is relevant, involving about $20 \%$ of women with clinical SUI. Although the correspondence between clinical and urodynamic diagnosis was high, we demonstrated that UDI may help in some cases to avoid an inappropriate surgical treatment. Therefore, UDI prior to SUI surgery should be considered to achieve a correct diagnosis and a proper therapeutic strategy.

\footnotetext{
* Correspondence: emanuele.rubilotta@aovr.veneto.it

'Department of Urology, Azienda Ospedaliera Universitaria Integrata (AOUI),

Piazzale Aristide Stefani, 1, 37126 Verona, Italy

Full list of author information is available at the end of the article
}

(c) The Author(s). 2019 Open Access This article is distributed under the terms of the Creative Commons Attribution 4.0 International License (http://creativecommons.org/licenses/by/4.0/), which permits unrestricted use, distribution, and reproduction in any medium, provided you give appropriate credit to the original author(s) and the source, provide a link to the Creative Commons license, and indicate if changes were made. The Creative Commons Public Domain Dedication waiver (http://creativecommons.org/publicdomain/zero/1.0/) applies to the data made available in this article, unless otherwise stated. 


\section{Background}

Uncomplicated stress urinary incontinence (SUI), also called pure stress urinary incontinence (PSUI), and the role of urodynamic investigation (UDI) prior to surgery for SUI have been under debate in the last years [1-3].

Since the exact prevalence of P-SUI is not clear, its clinical and economic impact is even not well defined. It has been calculated that in women with uncomplicated SUI and a confirmatory preoperative basic office evaluation, from 13 to 33 millions of US dollars could be saved annually by not performing UDI test [4]. Nevertheless, other authors reported that savings obtained by not performing UDI in these patients seem to be moderate [5].

Considering the cost of a single UDI, it has been debated if it is useful in women with uncomplicated SUI prior to surgical correction. Few papers showed that an office evaluation is a sufficient work-up for women with uncomplicated SUI, since their incontinence surgery outcomes were not inferior if compared to women who underwent UDI [6, 7]. However, the correlation between real prevalence of uncomplicated SUI and costs of clinical evaluation, UDI and surgical treatment has never been systematically analysed.

The aim of our study was to evaluate the prevalence of clinical P-SUI in a population of women who underwent UDI for urinary incontinence (UI), assessing the correspondence between clinical diagnosis of P-SUI and urodynamic stress incontinence. Our secondary end-point was to analyze the costs of UDI in women with P-SUI, and the number of surgical procedures that were believed unnecessary after UDI, including related money savings.

\section{Methods}

A single cohort of women who underwent UDI for UI between January 2012 and July 2016 was prospectively collected and retrospectively analyzed. Data were obtained from an electronic database. Urodynamic tests were performed according to the Good Urodynamic Practice [7]. P-SUI was defined by the International Continence Society (ICS) criteria. All women with clinical P-SUI had no history of: (i) previous pelvic surgical treatments; (ii) pain; (iii) aematuria; (iiii) recurrent infections; (iiiii) voiding symptoms; (iiiiii) pelvic irradiation; (iiiiiii) suspected fistula. Inclusion criteria were: naive patients for SUI without pelvic organ prolapse.

All women underwent UDI and their data were assessed by a urologist skilled in UDI and female urology. For all the patients, the first evaluation was obtained in the outpatient clinic recording patient's history and physical exam. Though the history, the clinician assessed the prevalent type of incontinence and its duration, moreover urinalysis and urine culture were analyzed. The physical exam included abdominal, pelvic and vaginal examination, a stress test (with an ultrasound estimated bladder volume of $200 \mathrm{ml}$, and if negative with a bladder volume of $300 \mathrm{ml}$ ) and uroflowmetry with post-voiding residual volume [8]. Then, in case of sterile urine culture, a UDI was performed. Finally, the correspondence between clinical and urodynamic stress incontinence was evaluated.

UDI findings were compared with clinical data and the results were considered different when a UDI outcome was not correspondent to the pre-UDI expected clinical data. Clinical P-SUI was not confirmed when at least one of the following UDI observations was found: i) mixed urinary incontinence with predominant detrusor overactivity; ii) detrusor underactivity; iii) voiding dysfunction. Patients were considered to have a detrusor underactivity when Qmax was $\leq 12 \mathrm{ml} / \mathrm{sec}$ and Pdet/ Qmax was $\leq 10 \mathrm{cmH} 2 \mathrm{O}$ [9]. Women were classified as having voiding dysfunction when Qmax was $\leq 12 \mathrm{ml} / \mathrm{sec}$ and Pdet/Qmax was $\geq 25 \mathrm{cmH} 2 \mathrm{O}[10,11]$. Depending on UDI results, surgical treatment options were discussed. Patients who had a change in the treatment strategy due to UDI results were recorded.

The exact cost of a single UDI was calculated with the support of the Clinical Management Department. The cost analysis included the human resources (one medical doctor and one nurse for each UDI) and all the materials used during the test. An additional economical evaluation was performed considering the National Health System reimbursement. Moreover, we calculated the total amount of expenses saved by not performing the scheduled surgical procedures of middle urethral sling (MUS) positioning, because they were believed unnecessary according to UDI results.

\section{Results}

Clinical SUI was diagnosed in 323/544 (59.4\%) patients. Prevalence of clinical P-SUI was 20.7\% (67/ 323), while prevalence of complicated SUI (C-SUI) was $79.3 \%(256 / 323)$.

After UDI, diagnosis of P-SUI decreased to 18.3\% (59/ 323). In $11.9 \%(8 / 67)$ of patients diagnosis of clinical P-SUI was not confirmed due to the detection of predominant detrusor overactivity (3 cases), detrusor underactivity (4 cases), and voiding dysfunction (1 case).

In $8.96 \%$ of cases $(6 / 67)$ the scheduled MUS was cancelled because of UDI results. In $3 / 6$ patients detrusor overactivity and urge incontinence were detected, in $2 / 6$ patients SUI was treated by pelvic floor rehabilitation, in $1 / 6$ case an important voiding dysfunction was detected. Results are better shown in Fig. 1, which reports patients characteristics based on clinical and UDI diagnoses and details about surgical UI correction cancelled after UDI. 


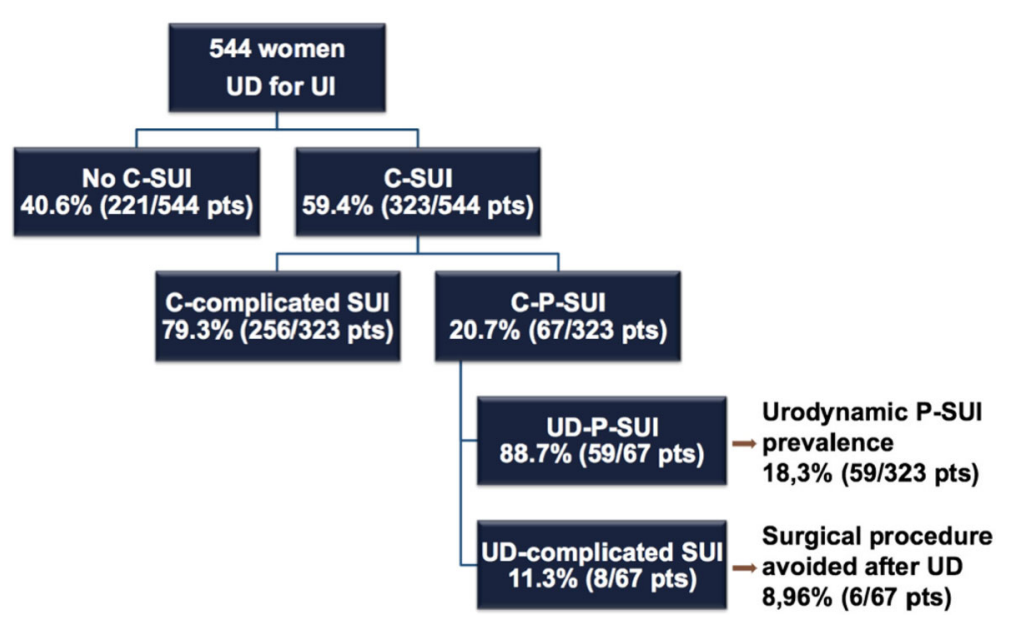

Fig. 1 Patients stratification based on clinical and urodynamic diagnoses, including their impact on surgical indications. (Legend: UDl: urodynamic investigation; UI, urinary incontinence; C-SUI: clinical stress urinary incontinence; P-SUI: pure stress urinary incontinence)

Being the Italian National Health System reimbursement for each UDI $296.5 €$, the total cost for the 59 uncomplicated patients in our series was $17,493.5 €$. If we consider a reimbursement by our National Health System ranging 1800-3073 € for each surgical procedure for female SUI (depending from the setting - Day Surgery or hospitalization for two or more days), the total amount saved thanks to UDI findings ranged $10,800-18,438 €$. These savings cover $61.7-105.0 \%$ of the costs for total number of UDIs performed in the uncomplicated patients in our series.

\section{Discussion}

In the literature few data are available on P-SUI with a prevalence ranging from 5.2 to $36 \%[1,2,12,13]$ (Table 1). In our series, prevalence was about $20 \%$, aligned with the results reported by Jeong [13].

Some authors believe that the high prevalence of P-SUI could be secondary to restricted clinical skills [3]. For this reason, our lower rate might be related to a higher expertise in the field of urodynamics and female urology. Furthermore, this know-how could also explain the high correspondence between UDI findings and clinical data which is a relevant controversial topic in the literature (Table 1).
Some patients experienced a change in their therapeutic strategy after UDI, leading to a significant reduction of unnecessary surgical procedures (8.96\%). Reversely, many women would have undergone an inappropriate surgical treatment (MUS) in case they did not perform UDI prior to surgery. Our results are aligned to those of Serati et al. who modified or cancelled the planned SUI surgery in 9.7 and $9.5 \%$ of cases, respectively [2].

In 2010, 260,000 MUS were implanted in the USA. If we consider a prevalence of clinical P-SUI of $20.7 \%$ (according to our study), we can therefore estimate that around 52,000 women underwent a MUS for P-SUI surgical correction [14]. Among these patients, almost 4659 (8.96\%) might have undergone an unnecessary surgery if the UDI had not been administered before surgical treatment.

Thus, the issue of unnecessary surgical procedures seems to represent a major clinical and economic topic. Re-intervention rates related to voiding dysfunction or to mesh extrusion have been reported in $3.7 \%$ of cases [15]. Although with the limitations of this analysis, if we match such data with our results, an estimated number of 172 women may have experienced these complications due to an unnecessary surgical

Table 1 Pure stress urinary incontinence (P-SUI) prevalence, correspondence between clinical PSUI and urodynamic findings, and avoided surgery after urodynamic testing

\begin{tabular}{llll}
\hline & P-SUI prevalence & Correspondence & Avoided surgery \\
\hline W. Agur et al., 2008 & $5.2 \%$ & - & - \\
S.J. Jeong et al., 2012 [13] & $20.7 \%$ & $79.1 \%$ & - \\
C.W. Nager et al., 2012 [1] & $34 \%$ & $43.2 \%$ & $5.4 \%$ \\
M. Serati et al., 2015 & $36 \%$ & $37.8 \%$ & $9.5 \%$ \\
E. Rubilotta et al., 2019 & $20.7 \%$ & $81.7 \%$ & $8.96 \%$ \\
\hline
\end{tabular}


treatment. Moreover, considering the reported chronic pain rate of $4.1 \%$ after MUS, the estimated number of patients with this potential complication after an unnecessary surgery would be around 191. Considering literature data, 740 mesh removal procedures have been reported in peer-reviewed publications and 7654 meshes were removed in patients presenting with sling complications, thus leading to a total of 8394 complicated women after MUS [15]. Just in order to extend the analysis, if we apply our criteria to the population of the cited study [14], we confirmed the previously reported results: 1738 patients were supposed to have P-SUI and in 156 of cases the SUI surgical intervention could have been cancelled after UDI. These results would be even higher if we refer to the greater P-SUI prevalence reported by the other authors $[1,2]$.

In the light of these considerations, we believe that UDI may help in the clinical diagnostic process of all women presenting with SUI and not only in the complicated ones. Indeed, UDI might help to rule out unnecessary surgical treatments and their potential sequelae.

Cost analysis must contemplate both direct and indirect costs. The direct costs comprehend the total amount for a single UDI, for the surgical procedure, and for hospitalization. The negative externalities are those costs that occur in the complicated patients, including repeated UDI, use of devices, and potentially a new surgical treatment and hospitalization. Our estimated rate of unnecessary surgical treatment was $8.96 \%$, meaning that without UDI these women would have had their insurance/health care to pay for their surgery without a confirmed indication. Nevertheless, insurance/health care would have paid even for the costs of the management of negative externalities.

In our study, we performed an analysis of the direct costs in a cohort of women with P-SUI. Analyzing the savings from the total number of UDIs performed in the uncomplicated patients in our series we found that costs of avoided surgical treatment covered the amount of money necessary for the UDIs. Regarding negative externalities, a complete cost evaluation should also include the potential legal actions due to complications after unnecessary interventions.

A limitation of our study may be related to the retrospective design, however all the data required were available for the analysis. A second limitation may be related to the different evaluations of the costs in diverse geographical areas.

\section{Conclusions}

The prevalence of clinical P-SUI is relevant, involving $1 /$ 5 of women with clinical SUI. The correspondence between clinical and urodynamic diagnosis is high.
Nevertheless, in some patients an inappropriate treatment was avoided basing on urodynamic results. Although UDI involves an economic burden, its role should be emphasized. Indeed, according to our data, UDI prior to SUI surgery is useful to avoid unnecessary surgical procedures and this finding should be taken into account when calculating the real costs/savings about performing or not UDI.

\section{Acknowledgements \\ None. \\ Funding \\ None. \\ Availability of data and materials \\ All datasets on which the conclusions of the manuscript rely are available from the corresponding author on reasonable request.}

\section{Authors' contributions}

$E R, M B$ designed the study, reviewed the literature and drafted the manuscript. SB, ADA, MAC participated in the design of the study, performed the procedures. CB performed the economic and statistical analysis. GV, PB participated in the study design and coordination. VI, DB participated in the study design and revised the manuscript. WA, EFA conceived of the study, participated in its design and coordination, supervised the draft. All authors read and approved the final manuscript.

\section{Ethics approval and consent to participate}

The research was based on a retrospective study, with participants selected on an internal database, and as such it was not considered necessary to submit for Institutional Review Board approval. Anyway, IRB of AOUI Verona was informed about our study. Principles of the Declaration of Helsinki were followed; the study was performed in compliance with Good Clinical Practice.

\section{Consent for publication}

A consent for publication was obtained from our patients.

\section{Competing interests}

EFA is a member of the Editorial Board (Associate Editor) of this journal. EFA was/is speaker honorarium/consultant for Laborie, Wellspect, Glaxo Smith Kline, Allergan, Coloplast, Astellas.

\section{Publisher's Note}

Springer Nature remains neutral with regard to jurisdictional claims in published maps and institutional affiliations.

\section{Author details}

'Department of Urology, Azienda Ospedaliera Universitaria Integrata (AOUI), Piazzale Aristide Stefani, 1, 37126 Verona, Italy. ${ }^{2}$ Healthcare Department Administrator, Azienda Ospedaliera Universitaria Integrata (AOUI), Verona, Italy. ${ }^{3}$ Department of Experimental Medicine and Surgery, Urology Unit, Tor Vergata University, Rome, Italy

Received: 13 August 2018 Accepted: 26 April 2019

Published online: 04 June 2019

\section{References}

1. Nager CW, Brubaker L, Litman HJ, Zyczynski HM, Varner RE, Amundsen C, Sirls LT, Norton PA, Arisco AM, Chai TC, Zimmern P, Barber MD, Dandreo KJ, Menefee SA, Kenton K, Lowder J, Richter HE, Khandwala S, Nygaard I, Kraus SR, Johnson HW, Lemack GE, Mihova M, Albo ME, Mueller E, Sutkin G, Wilson TS, Hsu Y, Rozanski TA, Rickey LM, Rahn D, Tennstedt S, Kusek JW, Gormley EA, Urinary Incontinence Treatment Network. A randomized trial of urodynamic testing before stress-incontinence surgery. N Engl J Med. 2012; 366(21):1987-97.

2. Serati M, Topazio L, Bogani G, Costantini E, Pietropaolo A, Palleschi G, Carbone A, Soligo M, Del Popolo G, Li Marzi V, Salvatore S, Finazzi Agrò E. 
Urodynamics useless before surgery for female stress urinary incontinence: are you sure? Results from a multicenter single nation database. Neurourol Urodyn. 2016;35(7):809-12.

3. Van Leiijsen SA, Kluivers KB, Mol BW, Broekhuis SR, Milani AL, Bongers MY, Aalders Cl, Dietz V, Malmberg GG, Vierhout ME, Heesakkers JP. Can preoperative urodynamic investigation be omitted in women with stress urinary incontinence? A non-inferiority randomized controlled trial. Neurourol Urodyn. 2012;31(7):1118-23.

4. Norton PA, Nager CW, Brubaker L, Lemack GE, Sirls LT, Holley R, Chai TC, Kraus SR, Zyczynski H, Smith B, Stoddard A. Urinary incontinence treatment Network. The cost of preoperative urodynamics: a secondary analysis of the ValUE trial. Neurourol Urodyn. 2016;35(1):81-4 Epub 2014 Oct 18.

5. Finazzi Agrò E, Perugia C, Topazio L, lacovelli V, Vespasiani G, Serati M. Urodynamic examinations performed before stress incontinence surgery in female patients: costs analysis in Italy. Neurourol Urodyn. 2016;35(issue S3): S51-2 Abstract n.52.

6. Serati M, Agrò EF. Urodynamics before surgery for stress urinary incontinence: the urodynamic examination is still one of the best friends of the surgeon and of patients with stress urinary incontinence. Eur Urol Focus. 2016;2(3):272-3.

7. Schafer W, Abrams P, Liao L, Mattiasson A, Pesce F, Spangberg A, Sterling AM, Zinner NR, van Kerrebroeck P. International continence society. Good urodynamic practices: uroflowmetry, filling cystometry, and pressure-flow studies. Neurourol Urodyn. 2002;21(3):261-74.

8. Guralnick ML, Fritel X, Tarcan T, Espuna M, Rosier PFWM. ICS-educational module: cough stress test in the evaluation of female urinary incontinence: introducing the ICS-uniform cough stress test. Neurourol Urodyn. 2018;37(5): 1849-55.

9. Gotoh M, Yoshikawa Y, Ohshima S. Pathophysiology and subjective symptoms in women with impaired bladder emptying. Int J Urol. 2006; 13(8):1053-7.

10. Defreitas GA, Zimmern PE, Lemack GE, Shariat SF. Refining diagnosis of anatomic female bladder outlet obstruction: comparison of pressure-flow study parameters in clinically obstructed women with those of normal controls. Urology. 2004;64(4):675-9 discussion 679-81.

11. Akikwala TV, Fleischman N, Nitti WW. Comparison of diagnostic criteria for female bladder outlet obstruction. J Urol. 2006:176(5):2093-7.

12. Agur W, Housami F, Drake M, Abrams P. Could the National Institute for health and clinical excellence guidelines on urodynamics in urinary incontinence put some women at risk of a bad outcome from stress incontinence surgery? BJU Int. 2009;103(5):635-9.

13. Jeong SJ, Kim HJ, Lee YJ, Lee JK, Lee BK, Choo YM, Oh JJ, Lee SC, Jeong CW, Yoon CY, Hong SK, Byun SS, Lee SE. Prevalence and clinical features of detrusor underactivity among elderly with lower urinary tract symptoms: a comparison between men and women. Korean J Urol. 2012;53(5):342-8.

14. Fabian G, Barcz E, Zwierzchowska A, Kociszewski J. Complications of suburethral sling procedures. Ginekol Pol. 2014;85:536-40.

15. Blaivas JG, Purohit RS, Benedon MS, Mekel G, Stern M, Billah M, Olugbade K, Bendavid R, lakovlev V. Safety considerations for synthetic sling surgery. Nat Rev Urol. 2015:12(9):481-509.

Ready to submit your research? Choose BMC and benefit from:

- fast, convenient online submission

- thorough peer review by experienced researchers in your field

- rapid publication on acceptance

- support for research data, including large and complex data types

- gold Open Access which fosters wider collaboration and increased citations

- maximum visibility for your research: over $100 \mathrm{M}$ website views per year

At $\mathrm{BMC}$, research is always in progress.

Learn more biomedcentral.com/submissions 